\title{
Existence of Multiple Solutions for a Class Non-uniformly Elliptic Equations with Critical Exponential Growth
}

\section{S. Khademloo' ${ }^{1}$ - M. Babagolzadeh ${ }^{1}$}

Received: 24 January 2018 / Revised: 28 April 2018 / Accepted: 18 May 2018 /

Published online: 11 June 2018

(C) The Author(s) 2018

Abstract This paper deals with the existence and multiplicity of weak solutions to non-uniformly elliptic problem

$$
-\operatorname{div}(a(x, \nabla u))+V(x)|u|^{N-2} u=\lambda\left(\exp \left(\alpha|u|^{\frac{N}{N-1}}\right)+f(x, u)\right),
$$

in $\mathbb{R}^{N}$, where $N \geq 2, \alpha$ is some positive constant and $\lambda$ is some positive parameter. Our method is mainly based on variational arguments.

Keywords $N$-Laplacian · Trudinger-Moser inequality · Critical exponential growth · Variational method $\cdot$ Local minima

Mathematics Subject Classification $35 \mathrm{~J} 25 \cdot 35 \mathrm{~J} 20 \cdot 35 \mathrm{~J} 61$

\section{Introduction}

In this paper, we are concerned with the existence and multiplicity of nontrivial weak solutions for the non-uniformly elliptic equations of $N$-Laplacian type of the form

$$
-\operatorname{div}(a(x, \nabla u))+V(x)|u|^{N-2} u=\lambda\left(\exp \left(\alpha|u|^{\frac{N}{N-1}}\right)+f(x, u)\right) \text { in } \mathbb{R}^{N},
$$

$凶$ S. Khademloo

s.khademloo@nit.ac.ir

M. Babagolzadeh

bs@nit.ac.ir

1 Department of Basic Sciences, Babol (Noshirvani) University of Technology, Babol, Iran 
where $N \geq 2, \alpha$ is some positive constant and $\lambda$ is some positive parameter, $V: \mathbb{R}^{N} \rightarrow$ ] $0,+\infty\left[\right.$ is a continuous function such that $V(x) \geq V_{0}$ for some positive constant $V_{0}$ and $V^{-1} \in L^{\frac{1}{N-1}}\left(\mathbb{R}^{N}\right)$ or $\lim V(x) \rightarrow \infty$ as $|x| \rightarrow \infty$, i.e., meas $\left\{x \in \mathbb{R}^{N}: V(x) \leq\right.$ $M\}<\infty$ for every $M>0$. Motivated by [6], we assume that $A$ be a measurable function on $\mathbb{R}^{N} \times \mathbb{R}$ such that $A(x, 0)=0$ and $a(x, \tau)=\frac{\partial A(x, \tau)}{\partial \tau}$ is a Carathéodory function on $\mathbb{R}^{N} \times \mathbb{R}$, and there are positive real numbers $c_{0}, c_{1}, k_{1}$ and two nonnegative measurable functions $h_{0}, h_{1}$ on $\mathbb{R}^{N}$ such that $h_{0} \in L^{N /(N-1)}\left(\mathbb{R}^{N}\right), h_{1} \in L_{\text {loc }}^{\infty}\left(\mathbb{R}^{N}\right)$ and $h_{1}(x) \geq 1$, satisfying the properties:

$\left(A_{1}\right)|a(x, \tau)| \leq c_{0}\left(h_{0}(x)+h_{1}(x)|\tau|^{N-1}\right), \forall \tau \in \mathbb{R}^{N}$, a.e. $x \in \mathbb{R}^{N}$,

$\left(A_{2}\right) 0 \leq a(x, \tau) . \tau \leq N A(x, \tau) \quad \forall \tau \in \mathbb{R}^{N}$, a.e. $x \in \mathbb{R}^{N}$,

$\left(A_{3}\right) \quad A(x, \tau) \geq k_{0} h_{1}(x)|\tau|^{N} \quad \forall \tau \in \mathbb{R}^{N}$, a.e. $x \in \mathbb{R}^{N}$.

Then $A$ verifies the growth condition:

$$
|A(x, \tau)| \leq c_{0}\left(h_{0}(x)|\tau|+h_{1}(x)|\tau|^{N}\right), \quad \forall \tau \in \mathbb{R}^{N} \text {, a.e. } x \in \mathbb{R}^{N} .
$$

Note that in the case of $N$-Laplacian, i.e., $A(x, \tau)=\frac{1}{N}|\tau|^{N}$, we choose

$$
a(x, \tau)=|\tau|^{N-2} \tau, \quad k_{0}=\frac{1}{N}, h_{1}(x)=1,
$$

which has been studied extensively, both in the case $N=2$ (i.e., Laplacian equation in $\mathbb{R}^{2}$ ) and in the case $N>3$ (i.e., $N$-Laplacian equation in $\mathbb{R}^{N}$ ).

The problems of this type are important in many fields of sciences, for example, in electromagnetism, astronomy and fluid dynamics. In fact, these models describe potentials of electric, gravitation and fluid, respectively.

In the case $p<N$, by the Sobolev embedding, the critical exponent is $p^{*}=\frac{p N}{N-p}$. When $p=N$, one has another maximal growth for any bounded domain $\Omega$, i.e.,

$$
\sup _{u \in W_{0}^{1, N}(\Omega),\|\nabla u\|_{L^{N} \leq 1}} \frac{1}{|\Omega|} \int_{\Omega} \exp \left(\alpha_{N}|u|^{N /(N-1)}\right) \mathrm{d} x<\infty
$$

where $\alpha_{N}=N W_{N-1}^{\frac{1}{N-1}}$ and $W_{N-1}$ is the surface area of the unit sphere in $\mathbb{R}^{N}$. In this situation, the constant $\alpha_{N}$ is sharp.([1,4,7])

In the case of unbounded domain, if we replace $\|\nabla u\|_{L^{N}}$ in the supremum by the standard Sobolev norm, then the following expression (Trudinger-Moser inequality) can be derived

$$
\sup _{u \in W_{0}^{1, N}\left(\mathbb{R}^{N}\right),\|\nabla u\|_{L^{N}}^{N}+|| u \|_{L^{N}}^{N} \leq 1} \int_{\mathbb{R}^{N}}\left(\exp \left(\alpha|u|^{N /(N-1)}\right)-S_{N-2}(\alpha, u)\right) \mathrm{d} x=\Xi,
$$

where $\Xi \leq \infty$ if $\alpha \leq \alpha_{N}$ and $\Xi=\infty$ if $\alpha>\alpha_{N}$. In this expression, $S_{N-2}(\alpha, u)=$ $\sum_{k=0}^{N-2} \frac{\alpha^{k}}{k !}|u|^{\frac{k N}{N-1}}$ (see [5,9]).

Motivated by the Trudinger-Moser inequality, we consider the following assumptions on $f$ which allows us to treat the problem (1.1) variationally in a subspace of 
$W^{1, N}\left(\mathbb{R}^{N}\right)$. We assume that $f: \mathbb{R}^{N} \times \mathbb{R} \rightarrow \mathbb{R}$ be a Carathéodory function that $\forall(x, s) \in \mathbb{R}^{N} \times[0,+\infty], \quad f(x, s) \geq 0$, and

$|f(x, u)| \leq b_{1}|u|^{\beta_{1}}+b_{2}|u|^{\beta_{2}}\left(\exp \left(\alpha_{0}|u|^{\frac{N}{N-1}}\right)-S_{N-2}\left(\alpha_{0}, u\right)\right), \quad \forall(x, u) \in \mathbb{R}^{N} \times \mathbb{R}^{+}$,

for some $b_{1}, b_{2}, \alpha_{0}>0$ and $\beta_{1}, \beta_{2} \geq 0$.

Next, we introduce some notations

$$
E=\left\{u \in W_{0}^{1, N}\left(\mathbb{R}^{N}\right): \int_{\mathbb{R}^{N}} h_{1}(x)|\nabla u|^{N} \mathrm{~d} x+\int_{\mathbb{R}^{N}} V(x)|u|^{N}<\infty\right\},
$$

which since $V$ is positive and bounded from below, $E$ is a reflexive Banach space when endowed with the norm

$$
\|u\|_{E}=\left(\int_{\mathbb{R}^{N}}\left(h_{1}(x)|\nabla u|^{N}+\frac{1}{k_{0} N} V(x)|u|^{N}\right) \mathrm{d} x\right)^{1 / N} .
$$

Then for $q \in[N,+\infty[$

$$
E \hookrightarrow W^{1, N}\left(\mathbb{R}^{N}\right) \hookrightarrow L^{q}\left(\mathbb{R}^{N}\right)
$$

with continuous embedding. Thus, there exists a positive constant $c_{2}$ such that

$$
\|u\|_{1, N} \leq c_{2}\|u\|_{E}, \quad \forall u \in E,
$$

where

$$
\|u\|_{1, N}=\left(\int_{\mathbb{R}^{N}}\left(|\nabla u|^{N}+|u|^{N}\right) \mathrm{d} x\right)^{\frac{1}{N}}
$$

is the norm in the Sobolev space $W^{1, N}\left(\mathbb{R}^{N}\right)$.

Using the radial lemma which asserts

$$
|u(x)| \leq|x|^{-1}\left(\frac{N}{W_{N-1}}\right)^{\frac{1}{N}}\|u\|_{N} \forall x \neq 0,
$$

for all $u \in W^{1, N}\left(\mathbb{R}^{N}\right)$ radially nonincreasing symmetric $([2,5])$, there exists a constant $c_{3}>0$ such that

$$
\int_{\mathbb{R}^{N}}\left[\exp \left(\alpha|u|^{\frac{N}{N-1}}\right)-S_{N-2}(\alpha, u)\right] \mathrm{d} x \leq c_{3}
$$

provided that $\|\nabla u\|_{N} \leq 1,\|u\|_{N} \leq M<\infty$ and $\alpha<\alpha_{N}$. Furthermore, there exists a constant $c_{4}>0$ such that

$$
\int_{\mathbb{R}^{N}}|u|^{q}\left[\exp \left(\alpha|u|^{\frac{N}{N-1}}\right)-S_{N-2}(\alpha, u)\right] \mathrm{d} x \leq c_{4}\|u\|_{1, N}^{q}
$$


provided that $\|u\|_{1, N} \leq M$ which $M<\left(\frac{\alpha_{N}}{\alpha}\right)^{\frac{N-1}{N}}$.

Inequalities (1.3) and (1.5) imply that there exists a constant $c_{5}>0$ such that

$$
\int_{\mathbb{R}^{N}}|u|^{q}\left[\exp \left(\alpha|u|^{\frac{N}{N-1}}\right)-S_{N-2}(\alpha, u)\right] \mathrm{d} x \leq c_{5}\|u\|_{E}^{q}
$$

provided that $\|u\|_{E} \leq M$ with $M<\frac{1}{c_{2}}\left(\frac{\alpha_{N}}{\alpha}\right)^{\frac{N-1}{N}}$.

Note that according to [9, Lemma 2.4], the assumptions $V^{-1} \in L^{1}\left(\mathbb{R}^{N}\right)$ or $\lim V(x) \rightarrow \infty$ as $|x| \rightarrow \infty$, on the potential $V(x)$ lead us to have the compactness of the imbedding $E \hookrightarrow L^{q}\left(\mathbb{R}^{N}\right)$, for all $1 \leq q<+\infty$.

Now from (1.2), the imbedding $E \hookrightarrow L^{q}\left(\mathbb{R}^{N}\right)$ for $q=\beta_{1}+1$ and (1.5) and (1.6) for $q=\beta_{2}+1$, there exist $c_{6}, c_{7}>0$ such that for all $(x, u) \in \mathbb{R}^{N} \times E$,

$$
\begin{aligned}
\int_{\mathbb{R}^{N}}|F(x, u)| \mathrm{d} x= & \int_{\mathbb{R}^{N}}\left|\int_{0}^{u(x)} f(x, s) \mathrm{d} s\right| \mathrm{d} x \\
\leq & \frac{b_{1}}{\beta_{1}+1} \int_{\mathbb{R}^{N}}|u|^{\beta_{1}+1} \mathrm{~d} x \\
& +\frac{b_{2}}{\beta_{2}+1} \int_{\mathbb{R}^{N}}|u|^{\beta_{2}+1}\left(\exp \left(\alpha|u|^{\frac{N}{N-1}}\right)-S_{N-2}(\beta, u)\right) \mathrm{d} x \\
\leq & \left.\frac{b_{1}}{\beta_{1}+1} c_{6}|| u\right|_{E} ^{\beta_{1}+1}+\frac{b_{2}}{\beta_{2}+1} c_{7} \|\left. u\right|_{E} ^{\beta_{2}+1}
\end{aligned}
$$

provided that $\|u\|_{E} \leq \frac{1}{c_{2}}\left(\frac{\alpha_{N}}{\alpha}\right)^{\frac{N-1}{N}}$.

Thus, by (1.4), we have $F(x, u) \in L^{1}\left(\mathbb{R}^{N}\right)$ for all $u \in E$. Therefore, the functional $I_{\lambda}: E \rightarrow \mathbb{R}$ given by

$$
I_{\lambda}(u)=\phi(u)-\lambda \psi(u),
$$

for

$$
\begin{aligned}
\phi(u) & =\int_{\mathbb{R}^{N}} A(x, \nabla u) \mathrm{d} x+\frac{1}{N} \int_{\mathbb{R}^{N}} V(x)|u|^{N} \mathrm{~d} x \\
& \geq \int_{\mathbb{R}^{N}} k_{0} h_{1}(x)|\nabla u|^{N}+\frac{1}{N} \int_{\mathbb{R}^{N}} V(x)|u|_{E}^{N} \\
& =k_{0}\|u\|^{N}
\end{aligned}
$$

and

$$
\psi(u)=\int_{\mathbb{R}^{N}}\left(\int_{0}^{u(x)} \exp \left(\alpha|s|^{\frac{N}{N-1}}\right) \mathrm{d} s+F(x, u)\right) \mathrm{d} x,
$$


is well defined. Moreover, $I_{\lambda}$ is a $C^{1}$ functional on $E$ and for $u, v \in E$

$$
\begin{aligned}
I_{\lambda}^{\prime}(u) v= & \int_{\mathbb{R}^{N}} a(x, \nabla u) \nabla v \mathrm{~d} x+\int_{\mathbb{R}^{N}} V(x)|u|^{N-2} u v \mathrm{~d} x \\
& -\lambda \int_{\mathbb{R}^{N}} \exp \left(\alpha|u|^{\frac{N}{N-1}}\right) v \mathrm{~d} x-\lambda \int_{\mathbb{R}^{N}} f(x, u) v \mathrm{~d} x .
\end{aligned}
$$

Consequently, the critical points of the functional $I_{\lambda}$ are precisely nontrivial weak solutions of the problem (1.1).

In the present paper, first we obtain the existence of a nontrivial weak solution for the problem (1.1), in a certain range of $\lambda$ (in Theorem 3.1). Then we prove the existence of the second weak solution for the problem (1.1) distinct from the first one (in Theorem 4.1).

\section{Preliminaries}

Our main tool is Theorem 2.2, consequence of a local minimum theorem [3, Theorem 5.1] which is inspired by Ricceri's variational principle.

For a given non-empty set $X$, and two functionals $\Phi, \Psi: X \rightarrow \mathbb{R}$, we define the following functions:

$$
\begin{aligned}
\varsigma\left(r_{1}, r_{2}\right) & =\inf _{v \in \Phi^{-1}(] r_{1}, r_{2}[)} \frac{\sup _{u \in \Phi^{-1}(] r_{1}, r_{2}[)} \Psi(u)-\Psi(v)}{r_{2}-\Phi(v)}, \\
\rho_{1}\left(r_{1}, r_{2}\right) & =\sup _{v \in \Phi^{-1}(] r_{1}, r_{2}[)} \frac{\Psi(v)-\sup _{u \in \Phi^{-1}(]-\infty, r_{1}[)} \Psi(u)}{\Phi(v)-r_{1}}
\end{aligned}
$$

for all $r_{1}, r_{2} \in \mathbb{R}, r_{1}<r_{2}$.

Definition 2.1 Let $\phi_{0}$ and $\psi_{0}$ be two continuously Gâteaux differentiable functionals defined on a real Banach space $X$ and fix $r_{1}, r_{2} \in[-\infty,+\infty]$, with $r_{1}<r_{2}$. We say that the functional $I_{0}=\phi_{0}-\psi_{0}$ verifies the Palais-Smale condition cut off lower at $r_{1}$ and upper at $r_{2}$ (in short ${ }^{\left[r_{1}\right]}(P S)^{\left[r_{2}\right]}$ ) if any sequence $\left(u_{n}\right) \subset X$ such that

(i) $\left(I_{0}\left(u_{n}\right)\right)$ is bounded,

(ii) if $I_{0}^{\prime}\left(u_{n}\right) \rightarrow 0$ in $X^{*}$ (where $X^{*}$ denotes the topological dual of $X$ ),

(iii) $r_{1}<\phi_{0}\left(u_{n}\right)<r_{2}, \forall n \in \mathbb{N}$

has a convergent subsequence. Clearly, if $r_{1}=-\infty$ and $r_{2}=+\infty$ it coincides with the classical $(P S)$ condition. Moreover, if $r_{1}=-\infty$ and $r_{2} \in \mathbb{R}$ it is denoted by $(P S)^{\left[r_{2}\right]}$.

Theorem 2.2 ([3] Theorem 5.1) Let $X$ be a real Banach space; $\Phi: X \rightarrow \mathbb{R}$ be a sequentially weakly lower semicontinuous, coercive and continuously Gâteaux differentiable function whose Gâteaux derivative admits a continuous inverse on $X^{*}$, $\Psi: X \rightarrow \mathbb{R}$ be a continuously Gâteaux differentiable function whose Gâteaux derivative is compact. Assume that there are $r_{1}, r_{2} \in \mathbb{R}, r_{1}<r_{2}$, such that

$$
\varsigma\left(r_{1}, r_{2}\right)<\rho_{1}\left(r_{1}, r_{2}\right)
$$


and for each $\lambda \in \Lambda=] \frac{1}{\rho_{1}\left(r_{1}, r_{2}\right)}, \frac{1}{\varsigma\left(r_{1}, r_{2}\right)}$ [ the functional $I_{\lambda}=\phi-\lambda \psi$ satisfies ${ }^{\left[r_{1}\right]}(P S)^{\left[r_{2}\right]}$ condition.

Then, for each $\lambda \in \Lambda$ there is $u_{0, \lambda} \in \Phi^{-1}(] r_{1}, r_{2}[)$ such that $I_{\lambda}\left(u_{0, \lambda}\right) \leq I_{\lambda}(u)$ $\forall u \in \Phi^{-1}(] r_{1}, r_{2}[)$ and $I_{\lambda}^{\prime}\left(u_{0, \lambda}\right)=0$.

Lemma 2.3 Assume that $\left(A_{1}\right),\left(A_{2}\right),\left(A_{3}\right)$ and $\left(f_{1}\right)$ hold. Then for each

$$
0<r<k_{0} \inf \left(1,\left(\frac{1}{2 c_{2}}\right)^{N}\left(\frac{(N-1) \alpha_{N}}{N \alpha_{0}}\right)\right)^{N-1}
$$

and $\lambda>0$, the functional $I_{\lambda}$ satisfies $(P S)^{[r]}$.

Proof Let $\left(u_{n}\right) \subset E$ be such that $\left(I_{\lambda}\left(u_{n}\right)\right)$ is bounded, $I_{\lambda}^{\prime}\left(u_{n}\right) \rightarrow 0$ and $\phi\left(u_{n}\right)<r$, for all $n \in \mathbb{N}$. Since $k_{0}\left\|u_{n}\right\|_{E}^{N} \leq \phi\left(u_{n}\right)$, so $\left\|u_{n}\right\|_{E}<\left(\frac{r}{k_{0}}\right)^{\frac{1}{N}}$. Therefore, there exists $u \in E$ such that $u_{n} \rightarrow u$ weakly in $E$. Thanks to Lemma 2.1. of [9] and using the assumption (1.2), one has

$$
\begin{aligned}
& \int_{\mathbb{R}^{N}}\left|f\left(x, u_{n}\right)\right|^{\frac{N}{N-1}} \mathrm{~d} x \\
& \leq b_{3}\left(\int_{\mathbb{R}^{N}}\left|u_{n}\right|^{\frac{\beta_{1} N}{N-1}} \mathrm{~d} x+\int_{\mathbb{R}^{N}}\left|u_{n}\right|^{\frac{\beta_{2} N}{N-1}}\left(\exp \left(\frac{N}{N-1} \alpha_{0}|u|^{\frac{N}{N-1}}\right)\right.\right. \\
& \left.\left.\quad-S_{N-2}\left(\frac{N \alpha_{0}}{N-1}, u\right)\right) \mathrm{d} x\right)
\end{aligned}
$$

where $b_{3}=\max \left\{b_{1}, b_{2}\right\}$. Since $\left\|u_{n}\right\|_{E} \leq\left(\frac{r}{k_{0}}\right)^{\frac{1}{N}}<\frac{1}{2 c_{2}}\left(\frac{\alpha_{N}}{\frac{N}{N-1} \alpha_{0}}\right)^{\frac{N-1}{N}}$, using (1.6) for $q=\frac{\beta_{2} N}{N-1}$, we deduce that

$$
\sup _{n \in \mathbb{N}}\left(\int_{\mathbb{R}^{N}} \mid f\left(x, u_{n}\right)^{\frac{N}{N-1}} \mathrm{~d} x\right)<+\infty .
$$

This fact together with the compactness of the imbedding $E \hookrightarrow L^{N}\left(\mathbb{R}^{N}\right)$ implies

$$
\lim _{n \rightarrow+\infty} \int_{\mathbb{R}^{N}} f\left(x, u_{n}\right)\left(u_{n}-u\right) \mathrm{d} x=0 .
$$

On the other hand, using the definition of $S_{N-2}$, one has

$$
\begin{aligned}
\int_{\mathbb{R}^{N}} \exp \left(\alpha\left|u_{n}\right|^{\frac{N}{N-1}}\right)\left(u_{n}-u\right) \mathrm{d} x= & \int_{\mathbb{R}^{N}} \exp \left(\alpha\left|u_{n}\right|^{\frac{N}{N-1}}-S_{N-2}\left(\alpha, u_{n}\right)\right)\left(u_{n}-u\right) \mathrm{d} x \\
& +\int_{\mathbb{R}^{N}} S_{N-2}\left(\alpha, u_{n}\right)\left(u_{n}-u\right) \mathrm{d} x .
\end{aligned}
$$

Using the compact imbedding $E \hookrightarrow L^{1}\left(\mathbb{R}^{N}\right)$ together with (1.4) for the first part of the above inequality and $E \hookrightarrow L^{2}\left(\mathbb{R}^{N}\right)$ together with the fact that $u_{n}$ is converges 
and so bounded in $L^{q}$ for any $q \geq 1$, arguing as before, one has

$$
\lim _{n \rightarrow+\infty} \int_{\mathbb{R}^{N}} \exp \left(\alpha\left|u_{n}\right|^{\frac{N}{N-1}}\right)\left(u_{n}-u\right) \mathrm{d} x=0
$$

Now using $\left(A_{1}\right)$, it follows that

$$
\begin{aligned}
\left|\left\langle I_{\lambda}^{\prime}\left(u_{n}\right), u_{n}-u\right\rangle\right|= & \mid \int_{\mathbb{R}^{N}}\left(a\left(x, u_{n}\right)\left(u_{n}-u\right)+V(x)\left|u_{n}-u\right|^{N-2}\left(u_{n}-u\right)\right) \mathrm{d} x \\
& -\lambda \int_{\mathbb{R}^{N}}\left(\exp \left(\alpha\left|u_{n}\right|^{\frac{N}{N-1}}\right)\left(u_{n}-u\right)+f\left(x, u_{n}\right)\left(u_{n}-u\right)\right) \mathrm{d} x \mid \\
\leq & \int_{\mathbb{R}^{N}}\left(\left|a\left(x, u_{n}\right)\right|\left|u_{n}-u\right|+V(x)\left|u_{n}\right|^{N-2}\left|u_{n}-u\right|\right) \mathrm{d} x \\
& +\lambda \int_{\mathbb{R}^{N}}\left(\left|\exp \left(\alpha\left|u_{n}\right|^{\frac{N}{N-1}}\right)\right|\left|u_{n}-u\right|+\left|f\left(x, u_{n}\right)\left(u_{n}-u\right)\right| \mathrm{d} x\right. \\
\leq & c_{0} \int_{\mathbb{R}^{N}}\left(\left(h_{0}(x)\left|u_{n}-u\right|+h_{1}(x)\left|u_{n}\right|^{N-1}\left|u_{n}-u\right|\right)\right. \\
& \left.+V(x)\left|u_{n}\right|^{N-2}\left|u_{n}-u\right|\right) \mathrm{d} x \\
& +\lambda \int_{\mathbb{R}^{N}}\left(\left|\exp \left(\alpha\left|u_{n}\right|^{\frac{N}{N-1}}\right)\right|\left|u_{n}-u\right|+\left|f\left(x, u_{n}\right)\left(u_{n}-u\right)\right| \mathrm{d} x .\right.
\end{aligned}
$$

This inequality together with $h_{0} \in L^{N /(N-1)}\left(\mathbb{R}^{N}\right)$ and $h_{1} \in L_{\text {loc }}^{\infty}\left(\mathbb{R}^{N}\right)\left(u_{n}\right.$ is converges to $u$ in $L^{1}$ and $L^{N}$ ) and using the fact

$$
\left\langle I_{\lambda}^{\prime}\left(u_{n}\right), u_{n}-u\right\rangle \rightarrow 0 \text { as } n \rightarrow+\infty
$$

it can be derived that $\left(u_{n}\right)$ is strongly convergent to $u$ in $E$.

\section{Existence of the First Solution}

Chosen large enough $R>0$ such that

$$
\begin{aligned}
& \text { (4) }{ }^{\frac{1+N}{N}} c_{0}\left(\int_{R \leq|x| \leq R+1} h_{0} \mathrm{~d} x\right)\left(C^{*}+\frac{b_{1}}{\beta_{1}+1} c_{6}+\frac{b_{2}}{\beta_{2}+1} c_{7}\right) \\
& <k_{0}^{\frac{1}{N}} B\left(k_{0} \int_{R \leq|x| \leq R+1} h_{1}(x) \mathrm{d} x+\frac{1}{N} \Gamma_{V}\right)^{\frac{N-1}{N}},
\end{aligned}
$$

where

$$
\Gamma_{V}=\int_{R \leq|x| \leq R+1} V(x) \mathrm{d} x+\int_{R \leq|x| \leq R+1} V(x)(R+1-|x|)^{N} \mathrm{~d} x
$$


and

$$
B=W_{N-1} \frac{R^{N}}{N}
$$

and the constant $c_{0}, c_{6}, c_{7}$ introduced in $\left(A_{1}\right),(1.6)$ and (1.7).

Now we are ready to present our first main result

Theorem 3.1 Assume that $\left(A_{1}\right),\left(A_{2}\right),\left(A_{3}\right)$ and $\left(f_{1}\right)$ hold. Then there exists $\lambda^{*}=$ $\lambda^{*}(r)>0$ such that for all $0<\lambda<\lambda^{*}$ the functional $I_{\lambda}$ admits a nontrivial critical point $u_{1}$ satisfying

$$
0<\phi\left(u_{1}\right)<r \text { and } I_{\lambda}\left(u_{1}\right) \leq I_{\lambda}(w) \text { for all } w \in \phi^{-1}(] 0, r[)
$$

for each $r>0$ with the property

$$
r \leq \frac{k_{0}}{4} \inf \left(1,\left(\frac{1}{c_{2}}\right)^{N}\left(\frac{(N-1) \alpha_{N}}{N \alpha_{0}}\right)^{N-1},\left(\frac{1}{c_{2}}\right)^{N}\left(\frac{\alpha_{N}}{\alpha}\right)^{N-1}\right)
$$

Proof For $\lambda>0$ and $R>0$ as in (3.1), define the function

$$
\theta_{\lambda}= \begin{cases}\eta_{\lambda} & \text { if }|x|<R \\ \eta_{\lambda}(R+1-|x|) & \text { if } R \leq|x| \leq R+1 \\ 0 & \text { if }|x|>R+1\end{cases}
$$

with $\eta_{\lambda}$ is a real number satisfying

$$
0<\eta_{\lambda}<\inf \left(Z_{\lambda}, T_{r}\right)
$$

where

$$
Z_{\lambda}=\left(\frac{\lambda B-c_{0} \int_{R \leq|x| \leq R+1} h_{0}}{c_{0} \int_{R \leq|x| \leq R+1} h_{1}+\frac{1}{N} \Gamma_{V}}\right)^{\frac{1}{N-1}}
$$

and

$$
T_{r}=\left(\frac{r}{k_{0} \int_{R \leq|x| \leq R+1} h_{1}(x)+\frac{1}{N} \Gamma_{V}}\right)^{\frac{1}{N}} .
$$

First we note that $\theta_{\lambda} \in E$.

Owing to our assumptions, one has

$$
\phi\left(\theta_{\lambda}\right)=\int_{\mathbb{R}^{N}} A\left(x, \nabla \theta_{\lambda}\right) \mathrm{d} x+\frac{1}{N} \int_{\mathbb{R}^{N}} V(x)\left|\theta_{\lambda}\right|^{N} \mathrm{~d} x
$$




$$
\begin{aligned}
& =\int_{R \leq|x| \leq R+1} A\left(x, \nabla \eta_{\lambda}(R+1-|x|)\right) \mathrm{d} x+\frac{1}{N} \int_{\mathbb{R}^{N}} V(x)\left|\theta_{\lambda}\right|^{N} \mathrm{~d} x \\
& \leq \int_{R \leq|x| \leq R+1} c_{0}\left(h_{0}(x)\left|\eta_{\lambda}\right|+h_{1}(x)\left|\eta_{\lambda}\right|^{N}\right) \mathrm{d} x+\frac{1}{N} \int_{\mathbb{R}^{N}} V(x)\left|\theta_{\lambda}\right|^{N} \mathrm{~d} x \\
& =c_{0} \eta_{\lambda} \int_{R \leq|x| \leq R+1} h_{0}(x) \mathrm{d} x+c_{0} \delta_{\lambda}^{N} \int_{R \leq|x| \leq R+1} h_{1}(x) \mathrm{d} x+\frac{1}{N} \delta_{\lambda}^{N} \Gamma_{V}:=G .
\end{aligned}
$$

So using the fact that $F\left(x, \theta_{\lambda}\right) \geq 0$, and $\exp \left(\alpha|s|^{\frac{N}{N-1}}\right) \geq 1$, it follows that

$$
\psi\left(\theta_{\lambda}\right) \geq \int_{|x|<R}\left(\int_{0}^{\theta_{\lambda}} \exp \left(\alpha|s|^{\frac{N}{N-1}}\right) \mathrm{d} s\right) \mathrm{d} x \geq \int_{|x|<R} \theta_{\lambda} \mathrm{d} x=B \eta_{\lambda} .
$$

Thanks to (3.2) and (3.3), we get

$$
\frac{\psi\left(\theta_{\lambda}\right)}{\phi\left(\theta_{\lambda}\right)} \geq \frac{B \delta_{\lambda}}{G} \geq \frac{1}{\lambda}
$$

On the other hand, if $u \in E$ has the property $\phi(u)<r$, then

$$
\begin{aligned}
k_{0} \int_{R \leq|x| \leq R+1} h_{1}(x)|\nabla u|^{N} \mathrm{~d} x & +\frac{1}{N} \int_{\mathbb{R}^{N}} V(x)|u|^{N} \mathrm{~d} x<\phi(u) \\
& =\int_{\mathbb{R}^{N}} A(x, \nabla u)+\frac{1}{N} \int_{\mathbb{R}^{N}} V(x)|u|^{N} \mathrm{~d} x<r,
\end{aligned}
$$

and so

$$
k_{0}\|u\|_{E}^{N} \leq \phi(u)<r
$$

i.e.,

$$
\|u\|<\left(\frac{r}{k_{0}}\right)^{\frac{1}{N}}
$$

By (3.1) and (1.7), there exists $C^{*}>0$ such that

$$
\begin{aligned}
\psi(u) & =\int_{\mathbb{R}^{N}}\left(\int_{0}^{u(x)} \exp \left(\alpha|s|^{\frac{N}{N-1}}\right) \mathrm{d} s+F(x, u)\right) \mathrm{d} x \\
\leq & \int_{\mathbb{R}^{N}}\left(\exp \left(\alpha|u|^{\frac{N}{N-1}}\right)|u| \mathrm{d} s+F(x, u)\right) \mathrm{d} x \\
\leq & \left.\int_{\mathbb{R}^{N}} \exp \left(\alpha|u|^{\frac{N}{N-1}}\right)-S_{N-2}(a, u)\right)|u| \mathrm{d} x \\
& +\int_{\mathbb{R}^{N}} S_{N-2}(a, u)|u| \mathrm{d} x+\int_{\mathbb{R}^{N}} F(x, u) \mathrm{d} x
\end{aligned}
$$




$$
\begin{aligned}
& \leq C^{*}\|u\|_{E}+\left(\frac{b_{1}}{\beta_{1}+1} c_{6}+\frac{b_{2}}{\beta_{2}+1} c_{7}\right)\|u\|_{E} \\
& \leq\left(C^{*}+\frac{b_{1}}{\beta_{1}+1} c_{6}+\frac{b_{2}}{\beta_{2}+1} c_{7}\right)\left(\frac{r}{k_{0}}\right)^{\frac{1}{N}} .
\end{aligned}
$$

Notice that in (3.5) we use the property $\|u\| \leq 1$.

Set

$$
\lambda^{*}=\frac{r^{\frac{N-1}{N}}}{\left(\frac{4}{k_{0}}\right)^{\frac{1}{N}}\left(C^{*}+\frac{b_{1}}{\beta_{1}+1} c_{6}+\frac{b_{2}}{\beta_{2}+1} c_{7}\right)} .
$$

Using the definition of $T_{r}$ and (3.4), we have $0<\phi\left(\theta_{\lambda}\right)<r$. Therefore, one has

$$
\begin{aligned}
\varsigma(0, r) & =\inf _{v \in \Phi^{-1}(] 0, r[)} \frac{\sup _{u \in \Phi^{-1}(] 0, r[)} \Psi(u)-\Psi(v)}{r-\Phi(v)} \\
& \leq \frac{\sup _{u \in \Phi^{-1}(] 0, r[)} \Psi(u)-\Psi\left(\theta_{\lambda}\right)}{r-\Phi\left(\theta_{\lambda}\right)} \\
& \leq \frac{\sup _{u \in \Phi^{-1}(] 0, r[)} \Psi(u)}{r} \leq \frac{1}{\lambda^{*}}<\frac{1}{\lambda}<\frac{\psi\left(\theta_{\lambda}\right)}{\phi\left(\theta_{\lambda}\right)} \\
& <\sup _{v \in \Phi^{-1}(] 0, r[)} \frac{\Psi(v)-\sup _{u \in \Phi^{-1}(]-\infty, 0[)} \Psi(u)}{\Phi(v)-0} \\
& =\rho_{1}(0, r),
\end{aligned}
$$

for all $0<\lambda<\lambda^{*}$. (Note that $\Psi(0)=0$ )

Hence, employing Theorem 2.2, for each $0<\lambda<\lambda^{*}$, the functional $\Phi-\lambda \Psi$ admits at least one critical point $u_{1} \in E$ such that $0<\phi\left(u_{1}\right)<r$, that is $\|u\|_{E}<\left(\frac{r}{k_{0}}\right)^{1 / N}$, and $I_{\lambda}\left(u_{1}\right) \leq I_{\lambda}(w)$, for any $w \in \phi^{-1}(] 0, r[)$. This completes the proof.

\section{Existence of the Second Solution}

Now, in this section, we prove the existence of the second local minimum distinct from the first one. For this purpose, we verify the hypotheses of Theorem 2.2. for another range of $r$.

Theorem 4.1 Assume that $\left(A_{1}\right),\left(A_{2}\right),\left(A_{3}\right)$ and $\left(f_{1}\right)$ hold. Then there exists $\lambda_{*} \in$ ]0, $\lambda^{*}$ [ such that for $\lambda_{*}<\lambda<\lambda^{*}$, the functional $I_{\lambda}$ admits a critical point $u_{2}$ which satisfies

$$
r<\phi\left(u_{2}\right)<2 r, \quad \text { and } I_{\lambda}\left(u_{2}\right) \leq I_{\lambda}(w), \quad \forall w \in \phi^{-1}(] r, 2 r[) .
$$

Proof First set

$$
\lambda_{*}=\left(\frac{2 r}{k_{0} \int_{R \leq|x| \leq R+1} h_{1}(x)+\frac{1}{N} \Gamma_{V}}\right)^{\frac{N-1}{N}}\left(\frac{2 c_{0} \int_{R \leq|x| \leq R+1} h_{0}}{B}\right) .
$$


From assumption (3.1), we get

$$
\lambda_{*}<\lambda^{*}
$$

For $\lambda_{*}<\lambda<\lambda^{*}$, we take the function $\theta_{\lambda}$ as before but in the following different condition:

$$
T_{r}<\eta_{\lambda}<\inf \left\{T_{2 r}, Z_{\lambda / 2}\right\}
$$

Therefore,

$$
r<k_{0} \int_{R \leq|x| \leq R+1} h_{1}(x)\left|\nabla \theta_{\lambda}\right|^{N} \mathrm{~d} x+\frac{1}{N} \int_{\mathbb{R}^{N}} V(x)\left|\theta_{\lambda}\right|^{N} \leq \phi\left(\theta_{\lambda}\right) \leq G<2 r,
$$

so 4.1 yields that

$$
\frac{\psi\left(\theta_{\lambda}\right)}{2 \phi\left(\theta_{\lambda}\right)}>\frac{B \eta_{\lambda}}{2\left(c_{0} \eta_{\lambda} \int_{R \leq|x| \leq R+1} h_{0}+c_{0} \delta_{\lambda}^{N} \int_{R \leq|x| \leq R+1} h_{1}+\frac{1}{N} \delta_{\lambda}^{N} \Gamma_{V}\right)}>\frac{1}{\lambda} .
$$

Similar to the arguments in the last section, by replacing $r$ by $2 r$, we have

$$
\begin{aligned}
\varsigma(r, 2 r) & =\inf _{v \in \Phi^{-1}(] r, 2 r[)} \frac{\sup _{u \in \Phi^{-1}(] r, 2 r[)} \Psi(u)-\Psi(v)}{2 r-\Phi(v)} \\
& \leq \frac{\sup _{u \in \Phi^{-1}(] r, 2 r[)} \Psi(u)-\Psi\left(\theta_{\lambda}\right)}{2 r-\Phi\left(\theta_{\lambda}\right)} \\
& \leq \frac{\sup _{u \in \Phi^{-1}(] r, 2 r[)} \Psi(u)}{2 r} \\
& \leq\left(\frac{1}{k_{0}}\right)^{\frac{1}{N}}\left(C^{*}+\frac{b_{1}}{\beta_{1}+1} c_{6}+\frac{b_{2}}{\beta_{2}+1} c_{7}\right)(2 r)^{-\frac{N-1}{N}} \\
& \leq\left(\frac{1}{k_{0}}\right)^{\frac{1}{N}}\left(C^{*}+\frac{b_{1}}{\beta_{1}+1} c_{6}+\frac{b_{2}}{\beta_{2}+1} c_{7}\right) r^{-\frac{N-1}{N}} \\
& <\frac{1}{\lambda^{*}}<\frac{1}{\lambda}<\frac{\psi\left(\theta_{\lambda}\right)-\sup _{u \in \Phi^{-1}(]-\infty, 0[)} \Psi(u)}{\phi\left(\theta_{\lambda}\right)-r} \\
& <\sup _{v \in \Phi^{-1}(] r, 2 r[)} \frac{\Psi(v)-\sup _{u \in \Phi^{-1}(]-\infty, r[)} \Psi(u)}{\Phi(v)-r} \\
& =\rho_{1}(r, 2 r), \quad
\end{aligned}
$$

for all $\lambda_{*}<\lambda<\lambda^{*}$. Note that in the second inequality we use the fact that if $\frac{t_{1}}{t_{2}}<s<\frac{t_{3}}{t_{4}}$, then $\frac{t_{1}-t_{3}}{t_{2}-t_{4}}<s$, and the following inequalities:

$$
\frac{\psi\left(\theta_{\lambda}\right)}{2 \phi\left(\theta_{\lambda}\right)}>\frac{1}{\lambda}>\frac{\sup _{\phi(u)<2 r} \psi(u)}{2 r}>\frac{\sup _{r<\phi(u)<2 r} \psi(u)}{2 r}
$$

for all $\lambda_{*}<\lambda<\lambda^{*}$. 
Since

$$
0<2 r<k_{0} \inf \left(1,\left(\frac{1}{2 c_{2}}\right)^{N}\left(\frac{\alpha_{N}}{\frac{N}{N-1} \alpha_{0}}\right)^{N-1}\right)
$$

then by Lemma 2.3 , the functional $I_{\lambda}$ satisfies ${ }^{[r]}(P S)^{[2 r]}$. Hence, employing Theorem 2.2 , we conclude that the functional $I_{\lambda}$ admits a critical point $u_{2}$ satisfying

$$
r<\phi\left(u_{2}\right)<2 r, \quad \text { and } I_{\lambda}\left(u_{2}\right) \leq I_{\lambda}(w), \quad \forall w \in \phi^{-1}(] r, 2 r[),
$$

for each $\lambda_{*}<\lambda<\lambda^{*}$. Since $\phi\left(u_{1}\right)<r$, then $u_{1} \neq u_{2}$.

Remark 4.2 To prove the existence of another local minimum distinct from the first and second ones, we can assume an algebraic condition on $f$ with the classical AmbrosettiRabinowitz condition: there exist $v>N$ and $R^{\prime}>0$ such that

$$
0<v F(x, t) \leq t f(x, t) \text { for all }|t|>R^{\prime}, x \in \mathbb{R}^{N}
$$

Clearly, the functional $\Phi-\lambda \Psi$ is of class $C^{1}$ and $(\Phi-\lambda \Psi)(0)=0$.

The first part of proof guarantees that $u_{2} \in E$ is a local nontrivial local minimum for $\Phi-\lambda \Psi$ in $E$. Therefore, there is $s>0$ such that

$$
\inf _{\left\|u-u_{2}\right\|=s}(\Phi-\lambda \Psi)(u)>(\Phi-\lambda \Psi)\left(u_{2}\right)
$$

So the condition $\left[8\right.$, Theorem $\left.2.2,\left(I_{1}\right)\right]$ is verified. Now choosing $u \neq 0$, from Ambrosetti-Rabinowitz condition, one has

$$
\begin{aligned}
(\Phi-\lambda \Psi)(t u) \leq & c_{0} t \int_{\mathbb{R}^{N}} h_{0}(x)|\nabla u| \mathrm{d} x+c_{0} t^{N} \int_{\mathbb{R}^{N}} h_{1}(x)|\nabla u|^{N} \mathrm{~d} x \\
& +\frac{t^{N}}{N} \int_{\mathbb{R}^{N}} V(x)|u|^{N} \mathrm{~d} x \\
& -\lambda \int_{\mathbb{R}^{N}}\left(\int_{0}^{t u} \exp \left(\alpha|s|^{\frac{N}{N-1}}\right) \mathrm{d} s+F(x, t u)\right) \mathrm{d} x \\
\leq & c_{0} t \int_{\mathbb{R}^{N}} h_{0}(x)|\nabla u| \mathrm{d} x+c_{0} t^{N} \int_{\mathbb{R}^{N}} h_{1}(x)|\nabla u|^{N} \mathrm{~d} x \\
& +\frac{t^{N}}{N} \int_{\mathbb{R}^{N}} V(x)|u|^{N} \\
& -\lambda t \int_{\mathbb{R}^{N}}|u| \mathrm{d} x-\lambda a_{3} t^{\nu} \int_{\mathbb{R}^{N}}|u|^{\nu} \mathrm{d} x+\lambda a_{4} \longrightarrow-\infty
\end{aligned}
$$

as $t \rightarrow \infty$, since $v>N$. So the condition [8, Theorem 2.2, $\left.\left(I_{1}\right)\right]$ is fulfilled.

Moreover, by standard computations, the functional $\Phi-\lambda \Psi$ satisfies (PS) condition. Hence, the classical theorem of Ambrosetti and Rabinowitz gives a critical point $u_{3}$ 
of $\Phi-\lambda \Psi$ such that $(\Phi-\lambda \Psi)\left(u_{3}\right)>(\Phi-\lambda \Psi)\left(u_{2}\right)$. So $u_{1}, u_{2}$ and $u_{3}$ are distinct weak solutions of the problem (1.1).

Open Access This article is distributed under the terms of the Creative Commons Attribution 4.0 International License (http://creativecommons.org/licenses/by/4.0/), which permits unrestricted use, distribution, and reproduction in any medium, provided you give appropriate credit to the original author(s) and the source, provide a link to the Creative Commons license, and indicate if changes were made.

\section{References}

1. Adimurthi, Yang, Y.: An interpolation of Hardly inequality and Trundinger-Moser inequality in $\mathbb{R}^{N}$ and its applications. Int. Math. Res. Not. 13, 2394-2426 (2010)

2. Berestycki, H., Lions, P.L.: Nonlinear scalar field equations, I. Existence of ground state. Arch. Ratl. Mech. Anal. 82, 313-346 (1983)

3. Bonanno, G.: A critical point theorem via the Ekeland variational principle. Nonlinear Anal. 75, 2992 3007 (2012)

4. do Ò, J.M.: N-Laplacian equation in $\mathbb{R}^{N}$ with critical growth. Abstr. Appl. Anal. 2, 301-315 (1997)

5. do Ó, J.M., Medeiros, E., Severo, U.: On a quasilinear nonhomogeneous elliptic equation with critical growth in $\mathbb{R}^{n}$. J. Differ. Equ. 246, 1363-1386 (2009)

6. Lam, N., Lu, G.: Existence and multiplicity of solutions to equations of N-Laplacian type with critical exponential growth in $\mathbb{R}^{N}$. J. Funct. Anal. 262, 1132-1165 (2012)

7. Moser, J.: A sharp form of an inequality by N. Trudinger. Indiana Univ. Math. J. 20, 1077-1092 $(1970 / 1971)$

8. Rabinowitz, P.H.: Minimax Methods in Critical Point Theory with Applications to Differential Equations. CBMS Reg. Conf. Ser. Math. 65, Amer. Mat. Soc., Providence (1986)

9. Yang, Y.: Existence of positive solutions to quasi-linear elliptic equations with exponential growth in the whole Euclidean space. J. Funct. Anal. 262, 1679-1704 (2012) 